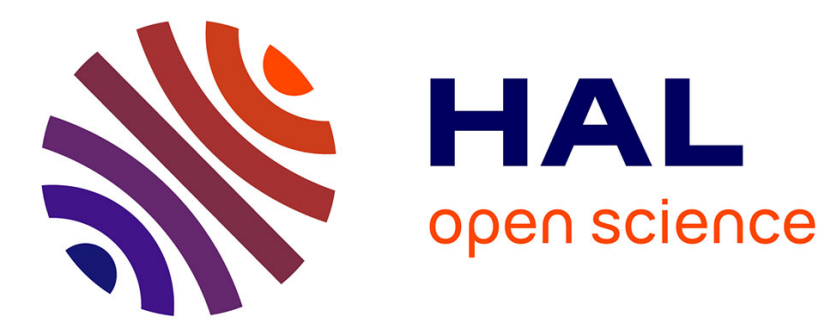

\title{
Users' Traces for Enhancing Arabic Facebook Search
}

Ismail Badache

\section{To cite this version:}

Ismail Badache. Users' Traces for Enhancing Arabic Facebook Search. The 30th ACM Conference on Hypertext and Social Media, Sep 2019, Hof, Germany. pp.241-245, 10.1145/3342220.3343661 . hal-02291127

\section{HAL Id: hal-02291127 \\ https://hal.science/hal-02291127}

Submitted on 18 Sep 2019

HAL is a multi-disciplinary open access archive for the deposit and dissemination of scientific research documents, whether they are published or not. The documents may come from teaching and research institutions in France or abroad, or from public or private research centers.
L'archive ouverte pluridisciplinaire HAL, est destinée au dépôt et à la diffusion de documents scientifiques de niveau recherche, publiés ou non, émanant des établissements d'enseignement et de recherche français ou étrangers, des laboratoires publics ou privés. 


\title{
Users' Traces for Enhancing Arabic Facebook Search
}

\author{
Ismail Badache \\ Aix Marseille Univ, Université de Toulon, CNRS, LIS, Marseille, France \\ Ismail.Badache@lis-lab.fr
}

\begin{abstract}
This paper proposes an approach on Facebook search in Arabic, which exploits several users' traces (e.g. comment, share, reactions) left on Facebook posts to estimate their social importance. Our goal is to show how these social traces (signals) can play a vital role in improving Arabic Facebook search. Firstly, we identify polarities (positive or negative) carried by the textual signals (e.g. comments) and non-textual ones (e.g. the reactions love and sad) for a given Facebook post. Therefore, the polarity of each comment expressed on a given Facebook post, is estimated on the basis of a neural sentiment model in Arabic language. Secondly, we group signals according to their complementarity using features selection algorithms. Thirdly, we apply learning to rank (LTR) algorithms to re-rank Facebook search results based on the selected groups of signals. Finally, experiments are carried out on 13,500 Facebook posts, collected from 45 topics in Arabic language. Experiments results reveal that Random Forests combined with ReliefFAttributeEval (RLF) was the most effective LTR approach for this task.
\end{abstract}

\section{CCS CONCEPTS}

- Information systems $\rightarrow$ Information retrieval;

\section{KEYWORDS}

Facebook Search, Sentiment Analysis, User Generated Content

\section{ACM Reference format:}

Ismail Badache. 2019. Users' Traces for Enhancing Arabic Facebook Search. In Proceedings of 30th ACM Conference on Hypertext and Social Media, Hof, Germany, September 17-20, 2019 (HT '19), 5 pages.

https://doi.org/10.1145/3342220.3343661

\section{INTRODUCTION}

Social media has largely contributed to the launch of the so-called Arab Spring. Since then, the penetration of social media has grown steadily. The number of Facebook users in the Arab world is estimated at 164 million $^{1}$. This movement reflects the democratization of the ways of production and interaction in the Web (usergenerated content) thanks to new technologies. Among these ways increasingly accessible to a wide audience include social networks, blogs, microblogs, etc. User-Generated Content (UGC) refers to a set

\footnotetext{
${ }^{1}$ https://arabiawithclass.com/164-million-active-facebook-users-in-the-arab-world-study-shows/
}

HT '19, September 17-20, 2019, Hof, Germany

https://doi.org/10.1145/3342220.3343661 of data (e.g. comments, posts, reactions) whose content is primarily either produced or directly influenced by end users. It can also be seen as a result of a computer-mediated communication [21].

The main task in information retrieval (IR) is to find a set of relevant documents to a specific information need (query). For this, effective approaches have existed for many years that exploit two classes of features to rank documents responding to a given query. The first class, the most used one, is query-dependent, which includes features corresponding to particular statistics of query terms such as term frequency, and term distribution within a document or in the collection of documents. The second class corresponds to query-independent features, which measure the a priori importance of the document. For example, number of backlinks [25], URL [32], PageRank [14], document authors [26] and social signals [6, 7].

This paper investigates the impact of users' traces (like, share, positive comment, negative comment, love, haha, angry, wow and sad) on the effectiveness of the relevance ranking of Arabic Facebook search. In order to design our approach, fundamental tasks are carried out. First, we identify the polarity for each comment left on a given post using a neural sentiment analysis in Arabic language. Then, we use feature selection algorithms to identify the most fruitful features (users' traces) for IR task. Finally, we evaluate the impact of these features on the relevance of Facebook search results. More specifically, we try to select the most effective features and combine them with Learning-To-Rank (LTR) approaches to improve IR on Facebook. The main contributions discussed in this paper are twofold:

(C1). Evaluate the impact of social features (users' traces and comment sentiment) on Facebook search. We try to answer the following research questions: a) What are the best social features suitable for this task? ; b) What is the impact of these features on the performance of Facebook's search ranking?

(C2). Build a test dataset (documents, topics, qrels) from Facebook. This dataset is useful to evaluate social IR systems in Arabic language. A user study is conducted to collect relevance judgments.

\section{BACKGROUND AND RELATED WORKS}

This section presents an overview of the Social Information Retrieval (SIR) and their major components related to our work. Beginning with a presentation of different types of UGCs, description and interrelationships of the Arabic sentiment analysis and our SIR approach. Then a focused overview of SIR approaches exploiting users' traces and social networks is presented.

User Generated Content is often linked to a specific social network with different operating rules (see table 1). The popularity of UGCs, especially in the context of social media, has given rise to many new problems in IR [13]. More specifically, how to exploit these social contents in favor of IR is an open question. 
Table 1: List of different types of UGCs (social signals)

\begin{tabular}{c|c|c}
\hline Type & Example & Social Networks \\
\hline Vote & Like, +1 & Facebook, LinkedIn, Google+, StumbleUpon \\
\hline Message & Tweet, Post & Facebook, Google+, LinkedIn, Twitter \\
\hline Share & Share, Re-tweet & Google+, Twitter, Buffer, Facebook, LinkedIn \\
\hline Tag & Bookmark, Pin & Delicious, Pinterest, Diigo, Digg \\
\hline Comment & Comment, Reply & Facebook, Google+, LinkedIn, Twitter \\
\hline Emotion & Love, Haha, Wow & Facebook \\
\hline Event Reaction & Thankful (Mother's day) & Facebook \\
\hline Relation & Followers, Friends & Facebook, Twitter \\
\hline
\end{tabular}

Arabic Sentiment Analysis is useful for quantifying the polarity of Arabic textual UGCs such as comments and tweets. However, to the best of our knowledge, just a few works have been done on sentiment analysis in Arabic language. This can be explained by the lack of standard datasets. Farra et al. [20] proposed a linguistic method based on a set of patterns to extract the polarities from a financial document. Al-Kabi et al. [2] have set up a tool that determines the subjectivity, the polarity of an opinion and its intensity. They used two general lexicons and sixteen specific lexicons. Abdulla et al. [1] proposed a statistical approach to detect subjectivity and polarity in social networks using morphological attributes. Bayoudhi et al. [12] compared three classifiers: SVM, Naive Bayes and a simple neural network. Ibrahim et al. [23] used a lexicon of 5244 adjectives, a lexicon of 3296 idioms to improve sentences classification with using SVM. Refaee and Rieser [31] applied a hybrid approach for predicting the intensity of polarity in tweets. They used logistic regression specifically to predict initial scores that are adjusted by applying rules extracted from a polarity lexicon. Other recent works apply deep learning techniques for opinion analysis [11,18]. Barhoumi et al. [11] used continuous representations of documents combined with a MultiLayer Perceptron (MLP) while Dahou et al. [18] used CNN (Convolutional Neural Network). Barhoumi et al. [10] illustrated a relevant comparison between several systems of Arabic sentiments detection, experienced in the Large-scale Arabic Book Review dataset (LABR $)^{2}$. They showed that the best results were obtained by Dahou et al. [18] using CNN (77.39\% of accuracy). The second best system is that of ElSahar and El-Beltagy [19], they have built a large Arabic lexicon multi-domains for sentiment analysis. The reviews was collected from various websites (e.g. hotels ${ }^{3}$, movies ${ }^{4}$ ). We recall that our goal in this paper is exploiting social features to improve Arabic Facebook search. For this, we used the approach proposed in [18] (see section 3.1) to measure comment polarity and consider it as an additional relevance factor.

Social Information Retrieval has extended traditional IR with different social features in order to satisfy social motivations behind the user's information needs. In 2012, Jaime Teevan ${ }^{5}$, a researcher at Microsoft, defines social IR as follows: "Social search is an emerging research area that explores how social interactions and social data can enhance existing information-seeking experiences, as well as enable new information retrieval scenarios. This session will showcase different models of social search, including 1) the use of social data to augment search,2) social data as new information to be searched, and 3) social interaction and collaboration as part of the search process."

\footnotetext{
${ }^{2}$ https://github.com/mohamedadaly/LABR

${ }^{3}$ https://www.tripadvisor.com/

${ }^{4} \mathrm{https://www.elcinema.com/}$

${ }^{5}$ https://www.microsoft.com/en-us/research/video/social-search-panel/
}

Our work concerns both the first and second axes mentioned by Jaime Teevan, we propose an approach to improve Facebook search using its UGCs. While considerable work has been done in the context of social IR in English language, there is still a lack of studies that would analyze the impact of users' traces on Facebook search in Arabic language. The most related works to ours include $[4,5,7,15,16,30]$. These works focus on the exploitation of social features to improve IR in English on the Web and on social networks. The approach we propose in this paper is in the same vein as these works, i.e exploiting social features around Facebook posts (documents) to improve the ranking of search results. However, our work differs from the state of the art in the following points. First, our approach is to search for information in Arabic language on Facebook. A sentiment analysis of the comments left by the users on a given publication is necessary. Next, we use Learning To Rank algorithms combined with feature selection techniques. More specifically, we estimate the social importance of a Facebook post by exploiting these social traces (like, share, polarity of comment: positive or negative, love, haha, angry, wow and sad) to improve the effectiveness of Arabic language search in Facebook.

\section{ARABIC FACEBOOK SEARCH APPROACH}

Our approach is based both on the classical traces (e.g. the frequencies of the signals "like" and "share", etc.) and on the emotional traces (e.g. the frequencies of the reactions "love" and "sad", etc.) as well as on the sentiment analysis of the comments expressed on each Facebook post (document). We note that Facebooks reactions allow users to express more nuanced emotions compared to classical signals. The goal of our approach is to improve the relevance of the results returned by the Facebook search engine in Arabic language by exploiting all these Facebook traces (or signals). They are considered as a priori knowledge to be taken into account in the Arabic Facebook search process.

\subsection{Social Traces-Based Search Process}

Three main steps are required: 1) extracting features and estimating sentiments for Arabic comments; 2) selecting the best features for IR task; and 3) combining LTR algorithms with selection techniques. The figure 1 illustrates our adopted Learning To Rank (LTR) process.

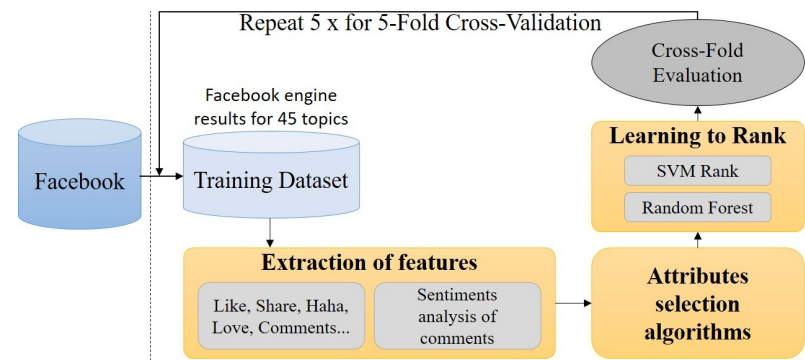

Figure 1: LTR process for Arabic Facebook search

Arabic Sentiment Analyzer. The sentiment of comment is estimated using the model proposed by Dahou et al. [18] whose implementation is publicly available ${ }^{6}$. Dahou et al. [18] proposed a $\mathrm{CNN}$ approach to identify the polarity of Arabic comments. When

\footnotetext{
${ }^{6}$ https://pan.baidu.com/s/1eS2mxCe\#list/path=\%2F
} 
considering the semantics of words, it has been shown that neural word embedding captures semantic similarities between the words [27]. Such distributed representations of words in a dense vector space are learned efficiently on large collections. Therefore, Dahou et al. [18] investigated different neural word embedding architectures using a corpus of 3.4 billion words chosen from a collected web-crawled corpus of 10 billion words. Then, the CNN was trained on top of the pre-trained word embeddings to classify the sentiments without considering aspect-level (topic on which the sentiment is concerned). They trained the model word2vec on web pages [27] using Skip-gram (SKIP-G) and Continuous Bag Of Words (CBOW) methods of constructing the training data for the neural network. Their experiments resultas showed that $\mathrm{CBOW}$ is more efficient and their architecture outperforms existing methods on several publicly available datasets presented in [3, 19, 29].

Selection of the Best Relevance Features. In this step, we relied on feature selection techniques to determine the best features groups that can be considered into the LTR of the IR process.

Combining LTR Algorithms with Selection Techniques. In this step, we studied the effectiveness of some feature selection techniques by confronting them with LTR algorithms. Since the performance of social features differs from one LTR algorithm to another, we identified the best feature selection techniques to find the best performing features according to the LTR algorithms.

\subsection{Facebook Data and Relevance Judgments}

To the best of our knowledge, there is no standard Arabic Facebook dataset containing posts, users' traces, topics and qrels to evaluate the effectiveness of Arabic IR on Facebook. Therefore, we collected 13,500 Arabic posts with their users' traces extracted from Facebook via its API and also using parsing, between 16 and 28 January 2018. These data were collected via the Facebook search engine for 45 topics that we have defined (Table 2 shows an example of Arabic topics). Table 3 presents statistics on the nature of the 45 topics. We note that we have exploited only public Facebook posts that are also open to indexing by search engines like Google or Bing. Unlike the Scandal Facebook-Cambridge Analytica ${ }^{7}$ where private and personal data were exploited for fraudulent purposes as for politicians. In our work any personal or private information has been exploited for our experimental evaluation. Table 4 gives some statistics about our dataset. It presents the 10 features we considered for estimating the relevance of Facebook posts for a given Arabic topic. The nature of the features from $f_{1}$ to $f_{10}$ is a simple count, for example the feature $f_{1}$ and $f_{4}$ represent the number of "Like" and emotional reaction "Sad", generated on the document. Concerning the last two features $f_{9}$ and $f_{10}$, they represent the number of opinions expressed on the document according to their polarity (positive or negative), respectively. These two features are calculated based on the Arabic sentiment model presented in 3.1.

Table 2: Examples of Arabic topics

\begin{tabular}{r|l}
\hline Arabic topic & Translation of the information need \\
\hline الطفل السوري عمران & $\begin{array}{l}\text { What happened to the Syrian child Omran, who was bombarded with his brother at } \\
\text { his home in Aleppo, and the global reaction to this tragedy. }\end{array}$ \\
\hline اضراب جامعة بيرزيت & $\begin{array}{l}\text { The reactions on the strike at Birzeit University (Palestine) and its impact on the } \\
\text { educational process. }\end{array}$ \\
\hline قتل السفير الروسي & $\begin{array}{l}\text { Why was the Russian ambassador killed in Turkey? And what are Arab and other } \\
\text { public opinions about his death? }\end{array}$ \\
\hline رقصة الزومبا & What are the benefits of Zumba and does it relate to weight loss? \\
\hline
\end{tabular}

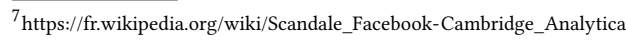

Table 3: Nature of the 45 topics

\begin{tabular}{c|c|c|c|c}
\multicolumn{5}{|c}{ Table 3: Nature of the } \\
\hline Politic & Sport & Art & Leisure & Other \\
\hline $42 \%$ & $24 \%$ & $18 \%$ & $11 \%$ & $5 \%$ \\
\hline
\end{tabular}

To obtain the relevance judgments for a given topic: 6 users were asked to assess the first 300 documents returned for a given topic using a 3-point relevance scale (irrelevant, somewhat relevant and relevant). Each topic is judged by 3 users. To avoid any bias, none of the social features were displayed with the documents, but all textual content, images or video (according to the Facebook post) are displayed to facilitate the task of judgment. We computed the agreement degree between assessors for each topic using Kappa Cohen measure $k$ [17]. The $k$ ranges from 0.45 to 0.90 . The average measure of agreement between the assessors is $75 \%$ (strong agreement).

Table 4: Facebook Data Statistics (list of exploited features)

\begin{tabular}{c|l|l|c|c|c|c}
\multicolumn{2}{c|}{ Posts (documents) \& Topics } & \multicolumn{1}{|c|}{ 13,500 documents } & \multicolumn{3}{|c}{ 45 Arabic topics } \\
\hline \hline$f_{i}$ & Feature & Description & SUM & MIN & MAX & AVG \\
\hline$f_{1}$ & Like & \#Like on the document & 2031958 & 0 & 32025 & 151 \\
$f_{2}$ & Share & \#Share on the document & 2329934 & 0 & 16781 & 173 \\
$f_{3}$ & Comment & \#Comment on the document & 2717589 & 0 & 24306 & 201 \\
$f_{4}$ & Sad & \#Sad on the document & 63970 & 0 & 80 & 5 \\
$f_{5}$ & Angry & \#Angry on the document & 95752 & 0 & 119 & 7 \\
$f_{6}$ & Love & \#Love on the document & 397679 & 0 & 496 & 29 \\
$f_{7}$ & Haha & \#Haha on the document & 246715 & 0 & 308 & 18 \\
$f_{8}$ & Wow & \#Wow on the document & 171234 & 0 & 213 & 13 \\
$f_{9}$ & Positive Comment & \#PositiveComment on the document & 1527546 & 0 & 13750 & 113 \\
$f_{10}$ & Negative Comment & \#NegativeComment on the document & 1134831 & 0 & 10063 & 84 \\
\hline
\end{tabular}

\subsection{Identification of the Most Effective Features}

In order to understand the real impact of the different social features, we evaluated the impact of each of them by using feature selection techniques. The goal is to determine the best features to exploit in the LTR algorithms. Feature selection techniques aim to identify and remove the maximum amount of unnecessary, redundant and irrelevant information upstream of a learning-based process [22]. They also make it possible to automatically select the subsets of features for obtaining the best results. We used Weka ${ }^{8}$ for these experiments, and we proceeded as follows: we identified relevant and irrelevant documents (posts) according to the "qrels", for the top 300 documents for each topic (45 Arabic topics) returned by the default Facebook search engine. The resulting set contains 13,500 documents including: 2971 relevant documents and 10529 irrelevant documents. We observed that this collection has an unbalanced relevance classes distribution. This occurs when there are many more elements in one class than in the other class of a training collection. In this case, a LTR algorithm usually tends to predict samples from the majority class and completely ignore the minority class. For this reason, we applied an approach to sub-sampling (reducing the number of samples that have the majority class) to generate a balanced collection composed of: 2971 relevant documents and 2971 irrelevant documents. Irrelevant documents for this study were selected randomly. Finally, we applied the selection algorithms on the two sets obtained, for 5 -folds cross-validation.

Feature selection algorithms consist in assigning a score to each feature according to its significance for the relevance class (relevant and irrelevant). These algorithms return importance ranking of the features according to the number of times that a given feature has been selected by the algorithm in the cross-validation. We note that we used for each algorithm the default setting provided by Weka.

\footnotetext{
${ }^{8}$ http://www.cs.waikato.ac.nz/ml
} 
Table 5: The selected features by the selection algorithms

\begin{tabular}{l|c|c|c|c|c|c|c|c|c|c}
\hline Algorithm & $f_{1}$ & $f_{2}$ & $f_{3}$ & $f_{4}$ & $f_{5}$ & $f_{6}$ & $f_{7}$ & $f_{8}$ & $f_{9}$ & $f_{10}$ \\
\hline CfsSubsetEval & + & + & - & - & - & + & - & + & + & + \\
\hline WrapperSubsetEval & + & + & - & - & - & + & - & - & + & + \\
\hline ConsistencySubsetEval & + & + & + & + & + & + & + & + & + & + \\
\hline FilteredSubsetEval & + & + & - & + & + & + & - & - & + & + \\
\hline ChiSquaredAttributeEval & + & + & + & + & + & + & + & + & + & + \\
\hline FilteredAttributeEval & + & + & + & + & + & + & + & - & + & + \\
\hline GainRatioAttributeEval & + & + & - & - & - & + & - & + & + & + \\
\hline InfoGainAttributeEval & + & + & + & + & + & + & + & + & + & + \\
\hline OneRAttributeEval & + & + & + & + & + & + & + & - & + & + \\
\hline ReliefFAttributeEval & + & + & - & + & + & + & + & + & + & + \\
\hline SVMAttributeEval & + & + & - & + & + & + & + & - & + & + \\
\hline SymetricalUncertEval & + & + & - & + & + & + & - & - & + & + \\
\hline Total & 12 & 12 & 5 & 9 & 9 & 12 & 7 & 6 & 12 & 12 \\
\hline
\end{tabular}

Table 5 presents the selected features by the 12 feature selection algorithms. A feature selected by the algorithm is a feature designated by a "+" and an unselected feature is designated by a "-". We remark that the features $f_{10}$ : Negative Comment, $f_{9}$ : Positive Comment, $f_{1}$ : Like, $f_{2}$ : Share and $f_{6}$ : Love are the most selected and height ranked compared to other features. The features $f_{4}$ : Sad, $f_{5}$ : Angry are moderately favored by the feature selection algorithms, except algorithms CfsSubsetEval, WrapperSubsetEval and GainRatioAttributeEval that did not selected them. The features $f_{7}$ : Haha, $f_{8}$ : Wow are only selected by 7 and 6 algorithms, respectively. Finally, the weakest and most disadvantaged feature is the $f_{3}$ : Comment, it is only selected by 5 out of 12 algorithms.

\subsection{Social Features-Based Learning to Rank}

Other experiments were carried out exploiting these social features in supervised approaches based on LTR models. We used the instances (Facebook posts) of the 45 topics as training sets. Then we used two LTR algorithms. This choice is explained by the fact that they often showed their effectiveness in IR: RankSVM [24] and Random Forests [28]. Regarding RankSVM, we use the implementation ${ }^{9}$ with its default settings proposed by Joachims [24]. While for Random Forests, we used Weka's implementation ${ }^{10}$. We have set the option "max depth" to 0 (unlimited) and the number of trees to 100 . The input of each algorithm is a vector of features (see table 4), that is all the features or only the features selected by a given selection algorithm. LTR algorithms predict the relevancy ranking of search results. Finally, we applied a cross-validation for 5-folds.

In order to take into account the selected social features in LTR models, we have carried out several experiments to identify the best features selection techniques allowing to find the most effective features according to the LTR techniques. Based on this study, we found the following best pairs of LTR algorithms and the feature selection techniques: a) Features selected by CfsSubsetEval (CFS) and WrapperSubsetEval (WRP) are learned using RankSVM and Random Forests; b) Features selected by ReliefFAttributeEval (RLF) are learned using Random Forests; and c) Features selected by SVMAttributeEval (SVM) are learned using RankSVM. We recall that features selection algorithms have highlighted 3 sets of features:

Table 6: Selected features sets (groups)

\begin{tabular}{l|l}
\hline Selection algorithms & Selected features \\
\hline CfsSubsetEval (CFS) & $f_{1}, f_{2}, f_{6}, f_{8}, f_{9}, f_{10}$ \\
WrapperSubsetEval (WRP) & $f_{1}, f_{2}, f_{6}, f_{9}, f_{10}$ \\
SVMAtrubuteEval (SVM) & $f_{1}, f_{2}, f_{4}, f_{5}, f_{6}, f_{7}, f_{9}, f_{10}$ \\
RelieffAttributeEval (RLF) & $f_{1}, f_{2}, f_{4}, f_{5}, f_{6}, f_{7}, f_{8}, f_{9}, f_{10}$ \\
\hline
\end{tabular}

In order to check the significance of the results compared to Facebook (baseline), we conducted the Student's t-test. We attached

\footnotetext{
${ }^{9} \mathrm{http} / / /$ www.cs.cornell.edu/people/tj/svm_light/svm_rank.html

${ }^{10} \mathrm{http} / / /$ weka.sourceforge.net/doc.dev/weka/classifiers/trees/RandomForest.html
}

* (strong significance) and ** (very strong significance) to the results in table 7 when $p$-value $<0.05$ and $p$-value $<0.01$, respectively.

Table 7: LTR results of $P @\{5,10\}$, nDCG et MAP

\begin{tabular}{|c|c|c|c|c|c|}
\hline \multicolumn{2}{|r|}{ IR Model } & $P @ 5$ & $\mathrm{P} @ 10$ & nDCG & MAP \\
\hline \multicolumn{2}{|c|}{ Facebook search engine (baseline model) } & 0.1911 & 0.1721 & 0.2513 & 0.1002 \\
\hline LTR Algorithms & Selection Algorithms & $\mathrm{P@5}$ & $\mathrm{P} @ 10$ & nDCG & MAP \\
\hline \multirow{4}{*}{ RankSVM } & CfsSubsetEval (CFS) & $0.2133^{*}$ & $0.1944^{*}$ & $0.2955^{*}$ & $0.1204^{*}$ \\
\hline & WrapperSubsetEval (WRP) & 0.1992 & 0.1802 & 0.2674 & 0.1076 \\
\hline & SVMAttributeEval (SVM) & $0.2627^{* *}$ & $0.2441^{* *}$ & $0.3939^{* * *}$ & $0.1654^{* *}$ \\
\hline & All features & $0.2254^{*}$ & $0.2066^{*}$ & $0.3196^{*}$ & $0.1314^{*}$ \\
\hline \multirow{4}{*}{ Random Forests } & CfsSubsetEval (CFS) & $0.2395^{*}$ & $0.2046^{*}$ & $0.2955^{*}$ & $0.1149^{*}$ \\
\hline & WrapperSubsetEval (WRP) & 0.2072 & 0.1883 & 0.2834 & 0.1149 \\
\hline & ReliefFAttributeEval (RLF) & $0.2920^{* * *}$ & $0.2735^{* *}$ & $0.4522^{* *}$ & $0.1921^{* * *}$ \\
\hline & All features & $0.2526^{* \prime \prime}$ & $0.2340^{*}$ & $0.3738^{\text {th }}$ & $0.1563^{*}$ \\
\hline
\end{tabular}

We now describe the results of our evaluation. We start with analyzing the effect of each features selection technique exploited with RankSVM and Random Forest algorithms. We then compare our results with those obtained by the baseline (Facebook search engine). Finally, we analyze the relative importance of each features selection technique according to their appropriated LTR algorithm.

Results obtained by RankSVM. The results obtained by RankSVM using the selection algorithm SVMAttributeEval (SVM), where only the two features $f_{3}$ and $f_{8}$ were not selected, are better than those obtained using (CFS, WRP or all features). We recorded improvement rates of $57 \%$ and $65 \%$ in terms of nDCG and MAP, respectively, compared to the baseline model. Using CFS which selects only 6 features $f_{1}, f_{2}, f_{6}, f_{8}, f_{9}, f_{10}$, and WRP which selects even fewer features $f_{1}, f_{2}, f_{6}, f_{9}, f_{10}$, the results fall with rates of $-25 \%$ and $-32 \%$ in terms of $\mathrm{nDCG}$, respectively. Consequently, the unselected features $f_{4}, f_{5}, f_{7}$ and $f_{8}$ are fruitful for RankSVM. In addition, with the selection of all features, RankSVM achieves better results than those obtained with CFS and WRP when certain features are ignored. Indeed, some topics such as (translation Arabic to English: the Syrian child Omran) and (translation Arabic to English: blockade of Gaza) recorded the highest precision when the features $f_{4}$ : Sad and $f_{5}$ : Angry are taken into account (with 0.8957 and 0.9324 in terms of P@10, respectively). The features $f_{8}$ : Wow and $f_{7}$ : Haha are more effective with topics that represent weird, exciting, or funny information. Finally, even if the RankSVM algorithm is expensive in terms of execution time, it remains favorable to obtain significant results. We noticed that RankSVM combined with the selection algorithm (SVM) obtained the second best result after the results obtained by Random Forests combined with the ReliefFAttributeEval (RLF) selection algorithm.

Results obtained by Random Forests. According to the table 7, the results confirm that the Random Forests decision tree is the most appropriate model when combined with the selection algorithm $R e$ liefFAttributeEval (RLF), it takes into account all the features, except for the feature $f_{3}$ : Comment, more efficiently than the other configurations (improvement rates of $80 \%$ and $92 \%$ in terms of nDCG and MAP compared to baseline model, respectively). The improvement rates compared to the baseline model using CFS and WRP are relatively low ( $18 \%$ and $13 \%$ in terms of $\mathrm{nDCG}$, respectively). We also note that Random Forests (combined with the RLF selection algorithm) exceeds the best RankSVM configuration (combined with the SVM selection algorithm) with a rate of $15 \%$ and $16 \%$ in terms of $\mathrm{nDCG}$ and MAP, respectively. In addition, the improvements are also highly significant for the configuration taking all the features with Random Forests (ranked $3^{r d}$ after Random Forest with RLF and RankSVM with SVM). 


\section{CONCLUSION}

To the best of our knowledge, we provide the first comprehensive investigation for the impact of the social features on Arabic Facebook search. This paper proposes a supervised approach of Arabic Facebook search based on social features specific to Facebook. Some features are a simple count (Like, Sad, Haha, etc.), while others represent a polarity of comments (positive or negative). We used feature selection techniques combined with learning to rank algorithms. The evaluation conducted on the Facebook dataset shows that Random Forests taking as input the features selected by RLF is the most successful configuration to estimate the relevance ranking of the results. In addition, LTR algorithms based on the most relevant features according to the selection algorithms are generally better compared to those obtained when the selection algorithms are ignored. Finally, we note that we are aware that the assessment of our approach is still limited. The main weakness of our approach is its dependence on the quality of the sentiment analysis model. An essential treatment step for an effective Arabic SIR is to use a stemmer for dialectal Arabic. Further large-scale experiments on other types of datasets are also envisaged. Even with these simple elements, the first results obtained encourage us to invest more in this track. As perspectives of this work in other context, we plan to adapt our approach to other types of information needs such as seeking controversial and contradictory information around specific topics, using pre-processing approaches on the detection of controversies and contradictions $[8,9]$.

\section{REFERENCES}

[1] Nawaf A. Abdulla, Mahmoud Al-Ayyoub, and Mohammed Naji Al-Kabi. An extended analytical study of arabic sentiments. IfBDI, 1(1/2):103-113, 2014.

[2] Mohammed N Al-Kabi, Amal H Gigieh, Izzat M Alsmadi, Heider A Wahsheh, and Mohamad M Haidar. Opinion mining and analysis for arabic language. IfACSA) International fournal of Advanced Computer Science and Applications, 5(5):181-195, 2014.

[3] Mohamed A. Aly and Amir F. Atiya. LABR: A large scale arabic book reviews dataset. In Proceedings of the 51st Annual Meeting of the Association for Computational Linguistics, ACL 2013, 4-9 August 2013, Sofia, Bulgaria, Volume 2: Short Papers, pages 494-498, 2013.

[4] Ismail Badache and Mohand Boughanem. Harnessing social signals to enhance a search. In 2014 IEEE/WIC/ACM International foint Conferences on Web Intelligence (WI) and Intelligent Agent Technologies (IAT), Warsaw, Poland, August 11-14, 2014 - Volume II, pages 303-309, 2014.

[5] Ismail Badache and Mohand Boughanem. A priori relevance based on quality and diversity of social signals. In Proceedings of the 38th International ACM SIGIR Conference on Research and Development in Information Retrieval, Santiago, Chile, August 9-13, 2015, pages 731-734, 2015.

[6] Ismail Badache and Mohand Boughanem. Emotional social signals for search ranking. In Proceedings of the 40th International ACM SIGIR Conference on Research and Development in Information Retrieval, Shinjuku, Tokyo, Japan, August 7-11, 2017, pages 1053-1056, 2017.

[7] Ismail Badache and Mohand Boughanem. Fresh and diverse social signals: Any impacts on search? In Proceedings of the 2017 Conference on Conference Human Information Interaction and Retrieval, CHIIR 2017, Oslo, Norway, March 7-11, 2017, pages 155-164, 2017.

[8] Ismail Badache, Sébastien Fournier, and Adrian-Gabriel Chifu. Finding and quantifying temporal-aware contradiction in reviews. In Information Retrieval Technology - 13th Asia Information Retrieval Societies Conference, AIRS 2017, Jeju Island, South Korea, November 22-24, 2017, Proceedings, pages 167-180, 2017.

[9] Ismail Badache, Sébastien Fournier, and Adrian-Gabriel Chifu. Predicting contradiction intensity: Low, strong or very strong? In The 41st International ACM SIGIR Conference on Research \& Development in Information Retrieval, SIGIR 2018, Ann Arbor, MI, USA, July 08-12, 2018, pages 1125-1128, 2018.

[10] Amira Barhoumi, Nathalie Camelin, and Yannick Estève. Des représentations continues de mots pour l'analyse d'opinions en arabe: une étude qualitative. In 25e conférence sur le Traitement Automatique des Langues Naturelles (TALN 2018), 2018.

[11] Amira Barhoumi, Yannick Estève, Chafik Aloulou, and Lamia Hadrich Belguith. Document embeddings for arabic sentiment analysis. In Proceedings of the Firs
Conference on Language Processing and Knowledge Management, LPKM 2017, Kerkennah (Sfax), Tunisia, September 8-10, 2017., 2017.

[12] Amine Bayoudhi, Hatem Ghorbel, and Lamia Hadrich Belguith. Sentiment classification of arabic documents: Experiments with multi-type features and ensemble algorithms. In Proceedings of the 29th Pacific Asia Conference on Language, Information and Computation, PACLIC 29, Shanghai, China, October 30 - November 1, 2015, 2015.

[13] Mohamed Reda Bouadjenek, Hakim Hacid, and Mokrane Bouzeghoub. Social networks and information retrieval, how are they converging? A survey, a taxonomy and an analysis of social information retrieval approaches and platforms. Inf. Syst., 56:1-18, 2016.

[14] Sergey Brin and Lawrence Page. The anatomy of a large-scale hypertextual web search engine. Computer Networks, 30(1-7):107-117, 1998.

[15] Marco Buijs and Marco R. Spruit. The social score - determining the relative importance of webpages based on online social signals. In KDIR 2014 - Proceedings of the International Conference on Knowledge Discovery and Information Retrieval, Rome, Italy, 21 - 24 October, 2014, pages 71-77, 2014.

[16] Sergiu Chelaru, Claudia Orellana-Rodriguez, and Ismail Sengör Altingövde. How useful is social feedback for learning to rank youtube videos? World Wide Web, 17(5):997-1025, 2014.

[17] Jacob Cohen. A coefficient of agreement for nominal scales. Educational and psychological measurement, 20(1):37-46, 1960.

[18] Abdelghani Dahou, Shengwu Xiong, Junwei Zhou, Mohamed Houcine Haddoud, and Pengfei Duan. Word embeddings and convolutional neural network for arabic sentiment classification. In COLING 2016, 26th International Conference on Computational Linguistics, Proceedings of the Conference: Technical Papers, December 11-16, 2016, Osaka, Japan, pages 2418-2427, 2016.

[19] Hady ElSahar and Samhaa R. El-Beltagy. Building large arabic multi-domain resources for sentiment analysis. In Computational Linguistics and Intelligent Text Processing - 16th International Conference, CICLing 2015, Cairo, Egypt, April 14-20, 2015, Proceedings, Part II, pages 23-34, 2015.

[20] Noura Farra, Elie Challita, Rawad Abou Assi, and Hazem M. Hajj. Sentence-level and document-level sentiment mining for arabic texts. In ICDMW 2010, The 10th IEEE International Conference on Data Mining Workshops, Sydney, Australia, 13 December 2010, pages 1114-1119, 2010.

[21] Sandra Greiffenstern. The influence of computers, the internet and computermediated communication on everyday English. $\mathrm{PhD}$ thesis, Humboldt University of Berlin, 2010.

[22] Mark A. Hall and Geoffrey Holmes. Benchmarking attribute selection techniques for discrete class data mining. IEEE Trans. Knowl. Data Eng., 15(6):1437-1447, 2003.

[23] Hossam S. Ibrahim, Sherif M. Abdou, and Mervat Gheith. MIKA: A tagged corpus for modern standard arabic and colloquial sentiment analysis. In $2 n d$ IEEE International Conference on Recent Trends in Information Systems, ReTIS 2015, Kolkata, India, fuly 9-11, 2015, pages 353-358, 2015.

[24] Thorsten Joachims. Training linear svms in linear time. In Proceedings of the Twelfth ACM SIGKDD International Conference on Knowledge Discovery and Data Mining, Philadelphia, PA, USA, August 20-23, 2006, pages 217-226, 2006.

[25] Wessel Kraaij, Thijs Westerveld, and Djoerd Hiemstra. The importance of prior probabilities for entry page search. In SIGIR 2002: Proceedings of the 25th Annual International ACM SIGIR Conference on Research and Development in Information Retrieval, August 11-15, 2002, Tampere, Finland, pages 27-34, 2002.

[26] Craig Macdonald and Iadh Ounis. Voting for candidates: adapting data fusion techniques for an expert search task. In Proceedings of the 2006 ACM CIKM International Conference on Information and Knowledge Management, Arlington, Virginia, USA, November 6-11, 2006, pages 387-396, 2006.

[27] Tomas Mikolov, Kai Chen, Greg Corrado, and Jeffrey Dean. Efficient estimation of word representations in vector space. In 1st International Conference on Learning Representations, ICLR 2013, Scottsdale, Arizona, USA, May 2-4, 2013, Workshop Track Proceedings, 2013.

[28] Ananth Mohan, Zheng Chen, and Kilian Q. Weinberger. Web-search ranking with initialized gradient boosted regression trees. In Proceedings of the Yahoo! Learning to Rank Challenge, held at ICML 2010, Haifa, Israel, June 25, 2010, pages 77-89, 2011.

[29] Mahmoud Nabil, Mohamed A. Aly, and Amir F. Atiya. ASTD: arabic sentiment tweets dataset. In Proceedings of the 2015 Conference on Empirical Methods in Natural Language Processing, EMNLP 2015, Lisbon, Portugal, September 17-21, 2015, pages 2515-2519, 2015.

[30] Valeria Orso, Tuukka Ruotsalo, Jukka Leino, Luciano Gamberini, and Giulio Jacucci. Overlaying social information: The effects on users' search and information-selection behavior. Inf. Process. Manage., 53(6):1269-1286, 2017.

[31] Eshrag Refaee and Verena Rieser. ilab-edinburgh at semeval-2016 task 7: A hybrid approach for determining sentiment intensity of arabic twitter phrases. In Proceedings of the 10th International Workshop on Semantic Evaluation, SemEval@NAACLHLT 2016, San Diego, CA, USA, June 16-17, 2016, pages 474-480, 2016.

[32] Thijs Westerveld, Wessel Kraaij, and Djoerd Hiemstra. Retrieving web pages using content, links, urls and anchors. In Proceedings of The Tenth Text REtrieval Conference, TREC 2001, Gaithersburg, Maryland, USA, November 13-16, 2001, 2001. 